University of Nebraska - Lincoln

DigitalCommons@University of Nebraska - Lincoln

12-25-1995

\title{
Novel switching phenomena in ferroelectric Langmuir-Blodgett films
}

\author{
A. Bune \\ University of Nebraska - Lincoln \\ Stephen Ducharme \\ University of Nebraska - Lincoln, sducharme1@unl.edu \\ V.M. Fridkin \\ University of Nebraska - Lincoln \\ L. Blinov \\ Institute of Crystallography of the Russian Academy of Sciences, Moscow, Russia \\ S. Palto \\ Institute of Crystallography of the Russian Academy of Sciences, Moscow, Russia \\ See next page for additional authors
}

Follow this and additional works at: https://digitalcommons.unl.edu/physicsducharme

Part of the Physics Commons

Bune, A.; Ducharme, Stephen; Fridkin, V.M.; Blinov, L.; Palto, S.; Petukhova, N.; and Yudin, S., "Novel switching phenomena in ferroelectric Langmuir-Blodgett films" (1995). Stephen Ducharme Publications. 18.

https://digitalcommons.unl.edu/physicsducharme/18

This Article is brought to you for free and open access by the Research Papers in Physics and Astronomy at DigitalCommons@University of Nebraska - Lincoln. It has been accepted for inclusion in Stephen Ducharme Publications by an authorized administrator of DigitalCommons@University of Nebraska - Lincoln. 


\section{Authors}

A. Bune, Stephen Ducharme, V.M. Fridkin, L. Blinov, S. Palto, N. Petukhova, and S. Yudin 


\title{
Novel switching phenomena in ferroelectric Langmuir-Blodgett films
}

\author{
A. Bune, ${ }^{\text {a) }}$ Stephen Ducharme, ${ }^{\text {b) }}$ and V. Fridkin ${ }^{\text {a) }}$ \\ Department of Physics and Astronomy and the Center for Materials Research and Analysis, \\ University of Nebraska, Lincoln, Nebraska 68588-0111 \\ L. Blinov, S. Palto, N. Petukhova, and S. Yudin \\ Institute of Crystallography of the Russian Academy of Sciences, 117333 Moscow, Russia
}

(Received 13 September 1995; accepted for publication 31 October 1995)

\begin{abstract}
We have established ferroelectric switching and observed a novel conductance switching phenomenon in ferroelectric polymer films fabricated by the Langmuir-Blodgett technique. The films consist of 10-30 monolayers (ML) of a copolymer of vinylidene fluoride (70\%) and trifluoroethylene $(30 \%)$, exhibiting a first-order ferroelectric phase transition at $70{ }^{\circ} \mathrm{C}$ and nearly rectangular ferroelectric and conductance hysteresis at $24{ }^{\circ} \mathrm{C}$. Ferroelectric switching is accompanied by switching of the conductance by three orders of magnitude. A model describing this switching phenomenon is proposed. (C) 1995 American Institute of Physics.
\end{abstract}

Ferroelectric materials have fascinated scientists since their discovery in $1920 .{ }^{1,2}$ In analogy with ferromagnets, characteristic properties of ferroelectrics include a spontaneous electrical polarization, polarization reversal (switching), and disappearance of the polarization above a ferroelectric phase transition, or "Curie," temperature $T_{c}$. Ferroelectric polymers such as poly(vinylidene fluoride) and its copolymers, a special class of ferroelectric materials, have been the subject of intense study for over 25 years, yielding a wealth of physical data, ${ }^{3}$ and practical applications. ${ }^{4}$ The structure of a random copolymer of vinylidene fluoride and trifluoroethylene, $\mathrm{P}(\mathrm{VDF}-\mathrm{TrFE}){ }^{5,6}$ is shown in the inset of Fig. 1. The molecular units in the polymer chain have net dipole moments which point from the electronegative fluorine to the electropositive hydrogen. These chains crystallize in parallel rows with a rhombohedral $(\mathrm{mm} 2)$ point group symmetry and aligned dipoles in the ferroelectric state. ${ }^{7}$ Switching is accomplished by applying a large electric field to reverse the direction of polarization of all the chains.

One of the promising aspects of polymer ferroelectric materials is the possibility of preparing very thin films, for investigation of ferroelectric phenomena in low-dimension systems. The films have potential application in optical, electronic and sensor technologies. While films with thickness down to $600 \AA$ have been prepared by a spin coating technique, ${ }^{8}$ this technique is not suitable controlling the nanoscale structure. High quality thin films of ferroelectric $\mathrm{P}(\mathrm{VDF}-\mathrm{TrFE})$ were recently fabricated by our group ${ }^{9}$ using the Langmuir-Blodgett (LB) monolayer transfer technique, permitting precise control of the films nanostructure. ${ }^{10,11}$ Figure 1 shows the behavior of the capacitance during thermal cycling of a 30 ML LB film of P(VDF-TrFE) 70:30\% prepared as described previously. ${ }^{9}$ We assume that the LB technique deposits the films at a rate of $2 \mathrm{ML}$ per unit cell, the closest value consistent with the $\mathrm{P}(\mathrm{VDF}-\mathrm{TrFE})$ coverage on the water surface before transfer, ${ }^{9}$ leading to a calculated film

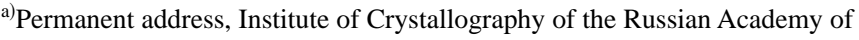
Sciences, 117333 Moscow, Russia.

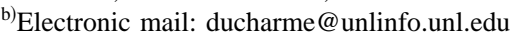

thickness of $7.4 \mathrm{~nm}$ (15 unit cells) for the 30-layer film. The film shows pronounced thermal hysteresis, a clear indication of the first-order ferroelectric phase transition. The ferroelectric Curie temperature of $70{ }^{\circ} \mathrm{C}$ is suppressed by $5{ }^{\circ} \mathrm{C}$ in the thin LB film compared to a much thicker film formed by solvent spinning.

Figure 2 shows the ferroelectric polarization switching, as probed by the pyroelectric response, ${ }^{12}$ in another 30-layer LB film. (We have used a pyroelectric probe of the spontaneous polarization instead of more direct methods, such as the Sawyer-Tower ${ }^{13}$ and the Merz methods, ${ }^{14}$ because of the large dc conductance.) The pyroelectric current was measured at zero bias voltage using a lock-in amplifier (with 30 ms time constant) as the sample was periodically heated by an $80-\mathrm{mW}$ laser beam (514.5 $\mathrm{nm}$ wavelength) chopped at $2000 \mathrm{~Hz}$. The hysteresis loops were formed beginning with a bias voltage of $+30 \mathrm{~V}$ (applied to the top electrode), decreas-

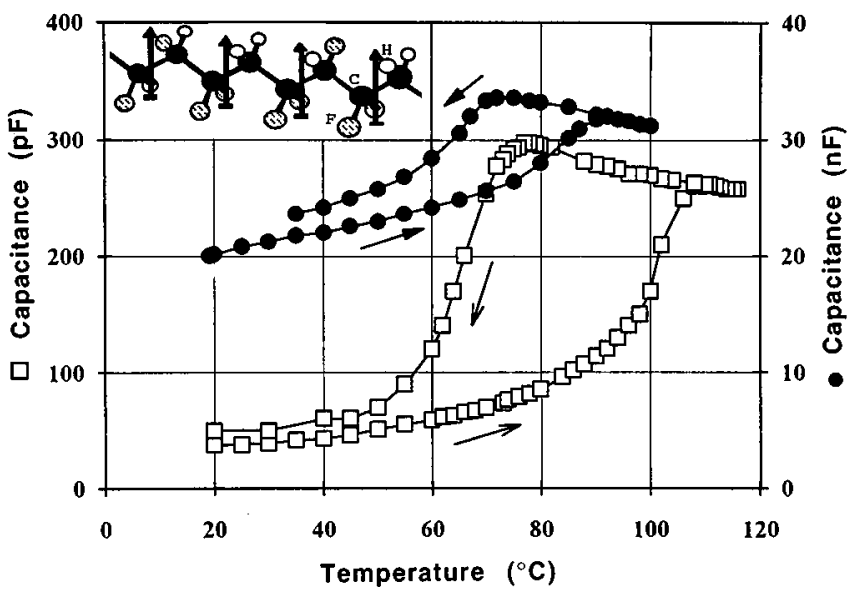

FIG. 1. Thermal hysteresis in the capacitance (in the dielectric constant) of the $\operatorname{VDF}(70 \%)-\operatorname{TrFE}(30 \%)$ ferroelectric copolymer. The $30 \mathrm{ML}$ LB film (solid circles) has a lower ferroelectric Curie temperature than a $15-\mu \mathrm{m}$ thick film made by solvent spinning (open squares). Inset: A fragment of the (VDF-TrFE) copolymer chain. $30 \%$ of the VDF $\left(-\mathrm{CH}_{2}-\mathrm{CF}_{2}-\right)$ units have been replaced at random by $\operatorname{TrFE}\left(-\mathrm{CHF}-\mathrm{CF}_{2}-\right)$ units. The arrows show the direction of the net dipole moments. 


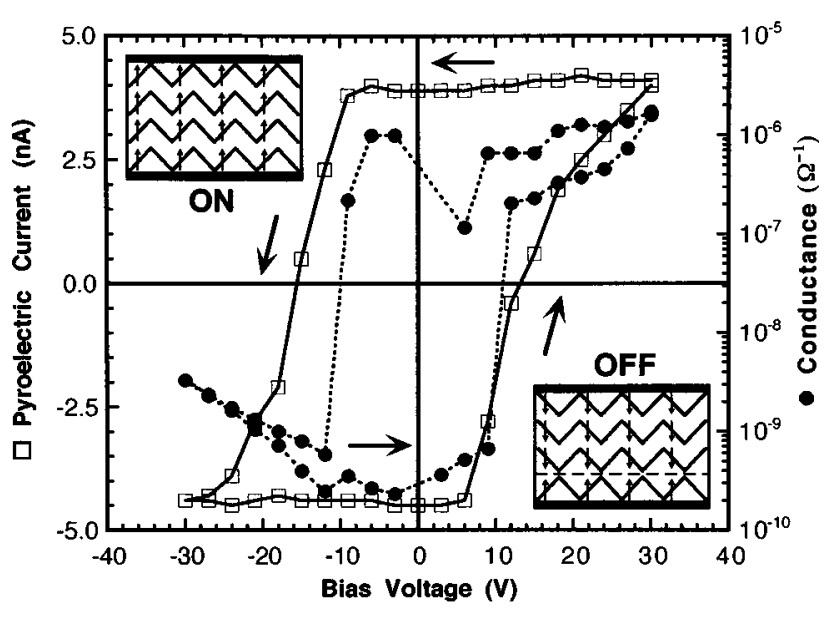

FIG. 2. Hysteresis in the pyroelectric current (open squares) and in the conductance (solid circles) of a $30 \mathrm{ML}$ ferroelectric Langmuir-Blodgett film of the $\operatorname{VDF}(70 \%)-\operatorname{TrFE}(30 \%)$ copolymer. Inset: Orientation of the polymer chains in the ON state of the fully ordered ferroelectric LB film (upper) and in the OFF state of the partially ordered LB film (lower).

ing in $3 \mathrm{~V}$ steps to $-30 \mathrm{~V}$, and back to $+30 \mathrm{~V}$. Each voltage level was maintained for $10 \mathrm{~min}$ after which the dc electrical conductance and transient pyroelectric current were recorded at zero voltage. The coercive field of $1.9 \pm 0.2 \times 10^{9} \mathrm{~V} / \mathrm{m}$ is forty times larger than the coercive field of the typical thick film made by solvent spinning. ${ }^{8}$ The generally rectangular shape of the polarization hysteresis implies that the LB films have a high degree of crystallinity. ${ }^{15}$ Figure 2 also shows a remarkable and unprecedented switching of the conductance, from very low conductance in the OFF state, obtained with negative polarization, to the 1000-times larger conductance in the ON state, obtained with positive polarization. The conductance switching has a slightly lower coercive field of $1.2 \pm 0.2 \times 10^{9} \mathrm{~V} / \mathrm{m}$ than the polarization switching. The hysteresis loops have been repeated over many cycles and several samples with various combinations of aluminum, indium tin oxide, and gold electrodes. The coincidence of the polarization and conductance hysteresis curves shown in Fig. 2 implies that the polarization controls the conductance mechanism.

The kinetics of switching offer further evidence for the connection between polarization and conductance as shown in Fig. 3. The conductance and pyroelectric currents were monitored while a $\pm 25 \mathrm{~V}$ switching voltage was applied to reverse a previously polarized state. The pyroelectric current was measured with the bias voltage on, resulting in an added contribution from the temperature derivative of the dielectric constant. (There is also an initial transient signal in both the pyroelectric current and the conductance current caused by circuit reactance.) The polarization switches to the ON state slowly [Fig. 3(a)] with a characteristic relaxation time of $3 \mathrm{~s}$ but the conductance switches OFF after a delay of $6 \mathrm{~s}$, when the polarization has reached $\sim 80 \%$ of its final value. In contrast, after application of a negative bias voltage the conductance switches OFF without apparent delay [Fig. 3(b)], but the polarization again switches slowly with a characteristic relaxation time of $2 \mathrm{~s}$. Measurements with greater time reso-

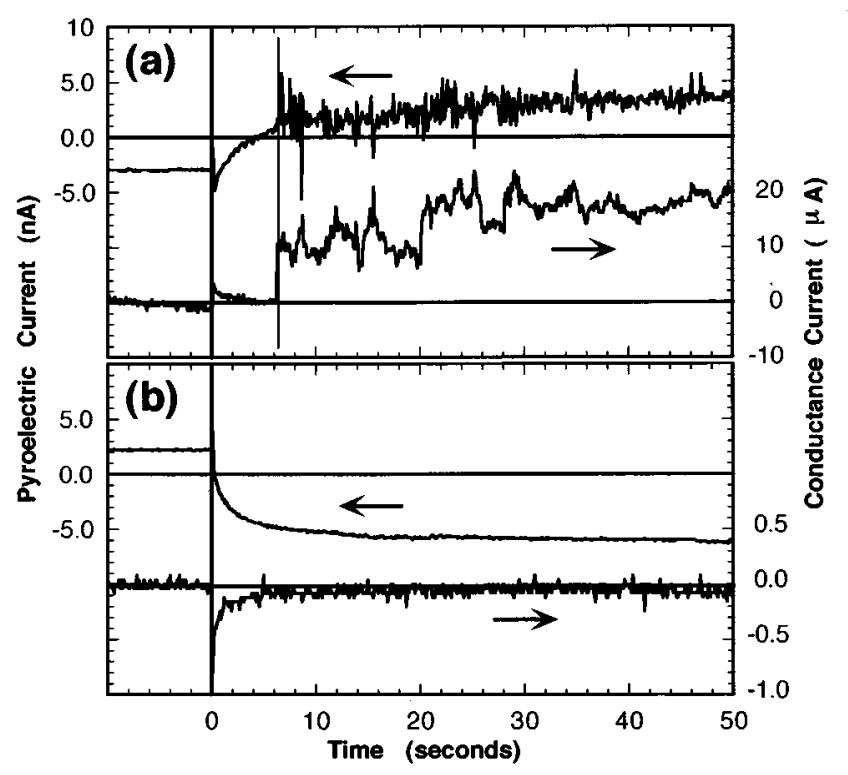

FIG. 3. The kinetics of polarization and conductance switching in a 30-layer Langmuir-Blodgett film of the ferroelectric $\operatorname{VDF}(70 \%)-\operatorname{TrFE}(30 \%)$ copolymer. (a) Switching ON. The sample is was first polarized in the OFF state with a $-25 \mathrm{~V}$ bias voltage for $15 \mathrm{~min}$. The negative bias voltage was removed and a positive $+25 \mathrm{~V}$ bias voltage applied at time $=0 \mathrm{~s}$. (b) Switching OFF. The sample is was first polarized in the ON state with a $+25 \mathrm{~V}$ bias voltage for $15 \mathrm{~min}$. The positive bias voltage was removed and a negative $-25 \mathrm{~V}$ bias voltage applied at time $=0 \mathrm{~s}$.

lution have determined that the duration of the conductance switching transition is less than $2 \mathrm{~ms}$, further indicating that conductance switching occurs upon reversal of a single monolayer which can occur in less than a nanosecond. ${ }^{16,17}$ Switching measurements at other voltages show that the polarization relaxation time strongly depends on the magnitude of the external electric field, but does not depend on its sign, as observed in films made by solvent spinning. ${ }^{18}$ The switching-ON transition of the conductance occurs only after the polarization has reached $\sim 80 \%$ of its saturated value while the switching-OFF transition occurs without significant delay.

The phenomenon of conductance switching has these important features: (1) It is connected with the bulk polarization switching; (2) there is a large (1000:1) contrast between the ON and OFF states; (3) the ON state is obtained only when the bulk polarization is switched in the positive direction; (4) the conductance switching is much faster than the bulk polarization switching. These main features are accomodated by the following model. Consider the arrangement (upper inset of Fig. 2) of a stack of fully polarized polymer chains extending from the substrate to the top electrode so that current can flow unimpeded from chain to chain as long as the dipoles are fully aligned. This is the ON state; current can flow through the film, perhaps controlled by tunneling, from the substrate to the top electrode. When the polarization is reversed, the majority of the layers reverse direction with the exception of one or a few layers that we assume are pinned to the upper electrode (and/or to the substrate) so that they cannot reverse direction (lower inset of Fig. 2). This is 
the OFF state; there is a barrier to charge transfer where the opposing dipoles meet at the domain boundary indicated by the dashed line. The conductance switches ON only after the 6-s delay, after the bulk polarization switching is nearly complete, when the last layer switches into alignment with the others, while the conductance switches OFF without a noticeable delay after the application of reverse bias as even one layer reverses and creates a barrier to charge transfer. The slow ( $\sim 2 \mathrm{~s}$ time constant) polarization switching shown in Fig. 3 is probably nucleation limited as has been observed in high-quality bulk films with low nucleation site densities. ${ }^{19}$ The duration of the conductance switching transition $(<2 \mathrm{~ms})$ may be limited only by the much faster switching time of individual layers.

The present results provide many new insights into the fundamental physics of ferroelectric polymers. In particular, the conductance switching is completely new and has possibly revealed the switching of a single layer. Additional measreuments of the conductance as a function of film thickness, voltage, and temperature will test the switching model and the conductance mechanism and will also provide a wealth of new information about ferroelectricity.

We emphasize that the nature of conductance switching in ferroelectric Langmuir-Blodgett films is completely new, and different from conductance switching which has been observed in other thin-film systems, e.g., polymers with conjugated bonds $^{20}$ or in amorphous semiconductors. ${ }^{21,22}$ The conductance switching phenomenon may result in a new kind of nonvolatile computer memories or data storage me- dia with simple nondestructive readout. The films also have potential application as optical or acoustical sensors.

${ }^{1}$ J. Valasek, Phys. Rev. 15, 537 (1920).

${ }^{2}$ M. E. Lines and A. M. Glass, Principles and Applications of Ferroelectrics and Related Materials (Clarendon, Oxford, 1977).

${ }^{3}$ T. Furukawa, Phase Trans. 18, 143 (1989).

${ }^{4}$ T. T. Wang, J. M. Herbert, and A. M. Glass, The Applications of Ferroelectric Polymers (Blackie, Glasgow, 1987).

${ }^{5}$ J. B. Lando and W. W. Doll, Macromol. Sci. Phys. B 2, 205 (1968).

${ }^{6}$ T. Yagi, M. Tatemoto, and J. Sako, Polym. J. 12, 209 (1980).

${ }^{7}$ A. J. Lovinger, G. T. Davis, T. Furukawa, and M. G. Broadhurst, Macromolecules 15, 323 (1982).

${ }^{8}$ K. Kimura and H. Ohigashi, Appl. Phys. Lett. 43, 834 (1983).

${ }^{9}$ S. Palto, L. Blinov, A. Bune, E. Dubovik, V. Fridkin, N. Petukhova, K. Verkhovskaya, and S. Yudin, Ferroelectric. Lett. 19, 65 (1995).

${ }^{10}$ L. M. Blinov, Sov. Phys. Usp. 31, 623 (1988).

${ }^{11}$ G. G. Roberts, Ferroelectrics 91, 21 (1989).

${ }^{12}$ A. G. Chynoweth, J. Appl. Phys. 27, 78 (1956).

${ }^{13}$ C. B. Sawyer and C. H. Towner, Phys. Rev. 35, 269 (1930).

${ }^{14}$ W. J. Merz, J. Appl. Phys. 27, 938 (1956).

${ }^{15}$ J. Jie, C. Bauer, B. Koslowski, and K. Dransfield, Physica B 204, 318 (1995).

${ }^{16}$ T. Furukawa, H. Matsuzaki, M. Shiina, and Y. Tajitsu, Jpn. J. Appl. Phys. 24, L661 (1985).

${ }^{17}$ H. Devy-Aharon, T. J. Sluckin, and P. L. Taylor, Phys. Rev. B 21, 3700 (1980).

${ }^{18}$ T. Furukawa and G. E. Johnson, Appl. Phys. Lett. 38, 1027 (1981).

${ }^{19}$ T. Furukawa, M. Date, M. Ohuchi, and A. Chiba, J. Appl. Phys. 56, 1481 (1984).

${ }^{20}$ K. Sakai, H. Matsuda, H. Kawada, K. Eguchi, and T. Nakagiri, Appl. Phys. Lett. 53, 1274 (1988).

${ }^{21}$ W. den Boer, Appl. Phys. Lett. 40, 812 (1982).

${ }^{22}$ S. R. Ovshinsky, Phys. Rev. Lett. 21, 1450 (1968). 